\title{
Concentrations of perfluoroalkyl and polyfluoroalkyl substances and blood glucose in pregnant women
}

Yanfeng Ren ${ }^{1+}$, Longmei Jin ${ }^{2 \dagger}$, Fen Yang ${ }^{3}$, Hong Liang ${ }^{4}$, Zhaofeng Zhang ${ }^{4}$, Jing Du ${ }^{4}$, Xiuxia Song ${ }^{4}$, Maohua Miao ${ }^{4^{*}}$ (D) and Wei Yuan ${ }^{4}$

\begin{abstract}
Background: Evidence on the association between exposure to perfluoroalkyl and polyfluoroalkyl substances (PFASs) and blood glucose concentrations in pregnant women is inconsistent. This study aimed to examine the association between PFAS exposure and the concentrations of fasting plasma glucose (FPG) and one-hour plasma glucose (1 h-PG) after a 50-g oral glucose tolerance test in pregnant women.

Methods: The study was based on the Shanghai-Minhang Birth Cohort, in which 1292 pregnant women were recruited. Among them, 981 women provided blood samples (at 12-16 gestational weeks) for PFAS measurement. FPG data collected from 856 women at 12-20 GW and 1 h-PG data collected from 705 women at 20-28 GW were obtained through medical records from the routine prenatal care system. High FPG or $1 \mathrm{~h}-\mathrm{PG}$ was defined as $\geq 90$ th percentile of FPG or $1 \mathrm{~h}-\mathrm{PG}$. The analysis of eight PFASs was conducted in this study: perfluorohexane sulfonate (PFHXS), perfluorooctane sulfonate (PFOS), perfluorooctanoic acid (PFOA), perfluorononanoic acid (PFNA), perfluorodecanoic acid (PFDA), perfluoroundecanoic acid (PFUdA), perfluorododecanoic acid (PFDoA), and perfluorotridecanoic acid (PFTrDA). The odds ratios (ORs) and associated 95\% confidence intervals (Cls) were estimated to determine the associations of each PFAS compound with high FPG and 1 h-PG from a logistic regression model.
\end{abstract}

Results: After adjustment for potential confounders, most PFASs were positively associated with high $1 \mathrm{~h}-\mathrm{PG}$ concentrations. The OR for high $1 \mathrm{~h}-\mathrm{PG}$ concentrations was 1.87 (95\% Cl: 1.15-3.05) with a one log unit increase of PFOS; similar associations were observed for PFNA (OR: 2.15, 95\% Cl: 1.24-3.74), PFDA (OR: 1.61, 95\% Cl: 1.10-2.44), PFUdA (OR: 1.71, 95\% Cl: 1.12-2.62), and PFDoA (OR: 1.34, 95\% Cl: 1.00-1.81). When the PFAS concentrations were categorized into three groups by tertiles, the highest tertiles of PFOS, PFOA, PFNA, PFDA, PFDoA, and PFTrDA had a statistically significant increase in the risk of high $1 \mathrm{~h}$ PG concentrations compared with the lowest tertiles. No statistically significant association was observed between PFAS exposure and high FPG.

Conclusion: PFAS exposure was associated with an increased risk of high 1 h-PG among pregnant women, but no such association was observed for FPG.

Keywords: Perfluoroalkyl and polyfluoroalkyl substances, Plasma glucose, Cohort study, Pregnancy

\footnotetext{
* Correspondence: miaomaohua@163.com

${ }^{\dagger}$ Yanfeng Ren and Longmei Jin contributed equally to this work.

${ }^{4} \mathrm{NHC}$ Key Lab of Reproduction Regulation (Shanghai Institute of Planned

Parenthood Research), Fudan University, Shanghai, China

Full list of author information is available at the end of the article
}

(c) The Author(s). 2020 Open Access This article is licensed under a Creative Commons Attribution 4.0 International License, which permits use, sharing, adaptation, distribution and reproduction in any medium or format, as long as you give appropriate credit to the original author(s) and the source, provide a link to the Creative Commons licence, and indicate if changes were made. The images or other third party material in this article are included in the article's Creative Commons licence, unless indicated otherwise in a credit line to the material. If material is not included in the article's Creative Commons licence and your intended use is not permitted by statutory regulation or exceeds the permitted use, you will need to obtain permission directly from the copyright holder. To view a copy of this licence, visit http://creativecommons.org/licenses/by/4.0/. The Creative Commons Public Domain Dedication waiver (http://creativecommons.org/publicdomain/zero/1.0/) applies to the data made available in this article, unless otherwise stated in a credit line to the data. 


\section{Introduction}

Perfluoroalkyl and polyfluoroalkyl substances (PFASs), a group of man-made chemicals with water-, stain-, and grease-resistant properties, are used in a wide range of consumer products, including fast food packaging, stainresistant carpets, windshield washing fluid, fire-fighting foam, insecticides, and paints [1]. Humans are widely exposed to PFASs through the ingestion of contaminated drinking water and food, as well as the inhalation of contaminated indoor air and dust [2]. Some PFASs have been shown to bio-accumulate in organisms [3]. The mean half-lives of PFASs in adult humans vary from 2.3 to 8.5 years $[4,5]$. The most commonly studied PFASs, including perfluorohexane sulfonate (PFHxS), perfluorooctane sulfonate (PFOS), perfluorooctanoate (PFOA), and perfluorononanoate (PFNA), are detected in the majority of human serum samples [6].

Animal studies have shown that PFAS exposure is associated with a wide range of adverse health effects, including the disruption of endocrine hormones, such as testosterone, estrogen, and thyroid hormones [7, 8], alterations in serum lipid levels [9], impaired glucose metabolism and insulin hypersensitivity [10], and immune system disturbance $[1,9]$. Human studies have also suggested the adverse effects of PFASs on the immune system [11], carcinogenesis [12], pregnancy-induced hypertension, arterial atherosclerosis [13, 14], and glucose metabolism [15-19].

Although epidemiological studies have suggested that PFASs are associated with impaired glucose tolerance and homeostasis, insulin resistance, beta-cell dysfunction, and a higher risk of diabetes [15-19], the associations observed in the general population cannot be generalized to metabolically vulnerable pregnant women owing to their special, insulin-resistant state during pregnancy. The current evidence on the effects of PFASs on glucose metabolism in pregnant women is limited and inconclusive. In the Odense Child Cohort study, PFHxS and PFNA concentrations were associated with impaired glycemic status in pregnant women and may therefore enhance the risk of developing gestational diabetes mellitus (GDM) [20]. In another prospective study of 258 women, higher pre-pregnancy PFOA concentrations were associated with an increased risk of GDM, but the associations for six other PFASs were not statistically significant [21]. In contrast, Valvi et al. found no associations between PFOA, PFOS, PFHxS, PFNA, or perfluorodecanoic acid (PFDA) concentrations and the risk of GDM in pregnant women [22].

In the present study, we sought to evaluate the associations between PFAS exposure and fasting plasma glucose (FPG) and 1-h plasma glucose concentrations (1 hPG) measured after a 50-g oral glucose tolerance test (OGTT) in pregnant women by using data from the Shanghai-Minhang Birth Cohort Study (S-MBCS).

\section{Methods}

\section{Study participants}

All study participants were recruited from the S-MBCS between April 2012 and December 2012. Pregnant women attending their first routine antenatal care at the Maternal and Child Health Hospital of Minhang district in Shanghai were consecutively recruited if: 1) they were at 12-16 gestational weeks (GW) of pregnancy; 2) they were registered residents of Shanghai; 3) they had no history of chronic disease of the liver, kidney, or other organs; 4) they planned to give birth in the study hospital; and 5) they were willing to participate in specified interviews during pregnancy and after delivery. Among 1670 pregnant women who were invited, 1292 pregnant women were recruited, corresponding to a response rate of $77.4 \%$.

\section{Exposure assessment and quality control}

Blood samples for PFASs measure were collected at recruitment, and plasma samples were separated and stored at $-80^{\circ} \mathrm{C}$, before they were transported to the Center for Disease Control and Prevention in Hubei Province for the assay of PFAS.

High-performance liquid chromatography coupled with tandem mass spectrometry (Agilent Technologies Inc., USA) was used for the quantitative measurement of PFASs. The information on sample collection, separation, reservation, transportation, quantification, limit of detection (LOD), and quality control has been detailed previously [6]. Among the 11 PFASs measured in our study, eight PFASs with detection rates above 90\%, including PFHxS, PFOS, PFOA, PFNA, PFDA, perfluorododecanoic acid (PFDoA), perfluoroundecanoic acid (PFUdA), and perfluorotridecanoic acid (PFTrDA), were included in the final analyses.

An internal standard approach was used to aid quantification. Milli-Q water was used to perform procedural blank analysis for each batch of samples. The concentrations of each detected congener should be more than three times of that in the procedural blank, and were corrected by subtracting the procedural blank concentration in the present study. LOD was defined as the concentration with a signal-tonoise ratio equal to or greater than 3 . All the recoveries ranged from $71.5-112.3 \%$. A five point calibration curve was drawn and each precursor ranged from $0.02-20.00 \mathrm{ng} / \mathrm{mL}$. Calibration curves presented a linear pattern over the concentration range of the precursor.

\section{Glucose and covariate measurement}

The information on plasma glucose concentrations in pregnant women was collected from the medical records of the prenatal care system, and included results for FPG 
and $1 \mathrm{~h}$-PG. In the study hospital within the study period, pregnant women were asked to provide blood after overnight fasting for FPG testing at their earliest conveniences, generally within 1 week after their first antenatal care. It was suggested that pregnant women underwent a $1 \mathrm{~h} \mathrm{50-g} \mathrm{OGTT} \mathrm{at} 20 \mathrm{GW}$ in order to screen for gestational diabetes, if they were considered to have a high risk of $\operatorname{GDM}(n=37)$, i.e., $\mathrm{FPG} \geq 6.1$ $\mathrm{mmol} / \mathrm{L}(110 \mathrm{mg} / \mathrm{dL})$ [23], or overweight and obese(i.e., $\mathrm{BMI} \geq 25 \mathrm{~kg} / \mathrm{m}^{2}$ ) [24]; otherwise, it was suggested that the examination of $1 \mathrm{~h}$-PG was performed between 24 and $28 \mathrm{GW}$. The 50-g OGTT was performed after an overnight fasting, also. The distribution of gestational weeks in which the FPG and $1 \mathrm{~h}$-PG examination was performed is shown in Supplemental Table S1. Information on whether the women had been diagnosed with GDM was extracted through medical records at birth.

A structured questionnaire was used by trained interviewers to collect information on the covariates. The women were asked about age, per capita household income, education level, passive smoking, height, prepregnancy weight, parity, history of abortion and stillbirth, pregnancy complications, etc. Pre-pregnancy BMI $\left(\mathrm{kg} / \mathrm{m}^{2}\right)$ was calculated as body weight divided by body height squared.

\section{Statistical analysis}

Among the 1292 pregnant women recruited, 1225 women delivered singleton live births, and 981 women provided blood samples at enrollment for PFAS measurement. FPG concentrations measured at 12-20 GWs were obtained for 856 women, and $1 \mathrm{~h}-\mathrm{PG}$ concentrations measured at 20-28 GWs were obtained for 705 pregnant women. Pregnant women who had data on PFASs and FPG concentrations were included in this study (Fig. 1). We first described and compared the demographic characteristics of the included and excluded pregnant women. The means and standard deviations (SD) were used to describe the distributions of FPG and $1 \mathrm{~h}-\mathrm{PG}$ according to the demographic characteristics of the included pregnant women. A logistic regression model was used to examine the association between PFAS exposure and plasma glucose, with the 90th percentiles of FPG $(4.6 \mathrm{mmol} / \mathrm{L}$, i.e., $83 \mathrm{mg} / \mathrm{dL})$ and $1 \mathrm{~h}-\mathrm{PG}(8.3 \mathrm{mmol} / \mathrm{L}$, i.e., $149 \mathrm{mg} / \mathrm{dL})$ used as the cutoff value. Natural logarithm (ln)-transformed PFAS concentrations were first included in logistic

\section{2 participants in the S-MBCS from} April 2012 to December 2012

Delivering in other hospital $(n=28) ;$
twin pregnancies $(n=8) ;$
abortions/stillbirths $(n=31)$

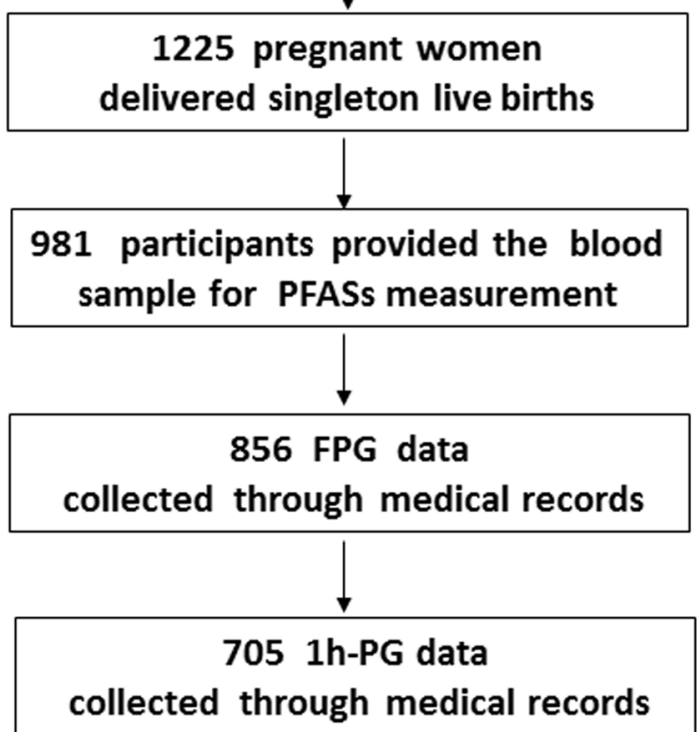

Fig. 1 Study population of the present study from S-MBCS. * FPG, fasting plasma glucose; 1 h-PG, 1 h-plasma glucose after a 50-g oral glucose tolerance test; S-MBCS, Shanghai-Minhang Birth Cohort Study 
regression models, and those with concentrations below the LOD were assigned a value of $\mathrm{LOD} / \sqrt{2}$. PFAS concentrations were also categorized into three groups by tertiles (T1: lowest tertile; T2: middle tertile; and T3: highest tertile) and included in the logistic regression models with the lowest tertile as the reference group. Odds ratios (ORs) and associated 95\% confidence intervals (CIs) were estimated for the association between each PFAS and high FPG/1 h-PG (i.e., $\geq 90$ th of FPG concentration or $\geq 90$ th of $1 \mathrm{~h}-\mathrm{PG}$ concentration). Based on tertiles, the concentrations were transformed to ordinal data and assigned to all persons to calculate $\mathrm{p}$-trend values. In addition, multiple linear regressions were used to analyze the association between PFASs and continuous plasma glucose concentrations.

Potential confounders were identified a prior according to the previous literature. Age of pregnant women, education, economic income, pre-pregnancy BMI, passive smoking, parity, history of abortion and stillbirth and pregnancy complications, including bleeding, thyroid disease and pregnancy-induced hypertension [20, 21, 25] were identified, and a directed acyclic graphs (Supplemental Figure S1) was used to evaluate the appropriation of covariates. We did not adjust for alcohol consumption $(n=6)$ in the final models because of the low prevalence. The statistical assumptions of logistic regressions were evaluated and met, including linear relationship of independent variables with logit(p), outliers, and colinearity of independent variables.

Several sensitivity analyses were performed to test the robustness of the primary results:1) Considering the potential effect of pre-pregnancy BMI on GDM [20] and the variation in PFAS concentrations across BMI, we repeated the analysis in women with a pre-pregnancy BMI of $<25 \mathrm{~kg} / \mathrm{m}^{2}$ to eliminate the confounding effect of BMI. 2) To test the generalizability of the results, we repeated the analyses in pregnant women without GDM. 3) To examine whether the associations of PFASs with FPG/1 h-PG were time-dependent, we performed subgroup analyses for different spans of GW at glucose measurement (for FPG, at 12-14 GWs and 15-20 GWs; for $1 \mathrm{~h}-\mathrm{PG}$, at 20-23 GWs and 24-28 GWs). Statistical Analysis System (SAS) software version 9.3 (SAS Institute, Inc., Cary, NC, USA) was used for statistical analysis. $P$ values of $<0.05$ were considered statistically significant.

\section{Results}

Table 1 presents the characteristics of the included pregnant women are compared with those of the excluded women in the study. The majority of women included in the present analyses were nulliparous (86.1\%), 25-35 years of age $(81.43 \%)$, with a BMI between $18.5-24.9 \mathrm{~kg} /$ $\mathrm{m}^{2}$ (74.17\%), with a household income per capita of >
Table 1 Characteristics of the included and excluded pregnant women

\begin{tabular}{|c|c|c|c|}
\hline Characteristics & $\begin{array}{l}\text { Included } \\
(N=856) \\
\mathrm{N}(\%) / \\
\text { Mean } \pm \mathrm{SD}\end{array}$ & $\begin{array}{l}\text { Excluded } \\
(N=369) \\
N(\%) / \\
\text { Mean } \pm \text { SD }\end{array}$ & $\begin{array}{l}P \text {-value of Student's } \\
\text { t-test or Chi-square test }\end{array}$ \\
\hline \multicolumn{4}{|c|}{ Maternal age at enrollment (years) } \\
\hline Mean \pm SD & $27.8 \pm 3.3$ & $27.8 \pm 3.4$ & 0.784 \\
\hline$<25$ & $128(15.0)$ & $56(15.2)$ & 0.454 \\
\hline $25-35$ & $697(81.4)$ & $294(79.7)$ & \\
\hline$\geq 35$ & $31(3.6)$ & $19(5.1)$ & \\
\hline \multicolumn{4}{|c|}{ Pre-pregnancy BMI $\left(\mathrm{kg} / \mathrm{m}^{2}\right)$} \\
\hline Mean \pm SD & $20.4 \pm 2.4$ & $20.5 \pm 2.4$ & 0.732 \\
\hline$<18.5$ & $176(20.9)$ & $68(18.7)$ & 0.666 \\
\hline $18.5-24.9$ & $627(74.6)$ & $279(76.6)$ & \\
\hline$\geq 25$ & $37(4.4)$ & $17(4.7)$ & \\
\hline \multicolumn{4}{|c|}{ Maternal education } \\
\hline $\begin{array}{l}\text { Below high } \\
\text { school }\end{array}$ & $76(8.9)$ & $43(11.7)$ & 0.314 \\
\hline High School & $125(14.6)$ & $50(13.5)$ & \\
\hline $\begin{array}{l}\text { College or } \\
\text { above }\end{array}$ & $653(76.5)$ & $276(74.8)$ & \\
\hline \multicolumn{4}{|c|}{ Per capita household income (CNY) } \\
\hline$<4000$ & $176(21.0)$ & $57(16.2)$ & 0.151 \\
\hline $4000-8000$ & $495(59.1)$ & $224(63.8)$ & \\
\hline$>8000$ & $167(19.9)$ & $70(19.9)$ & \\
\hline \multicolumn{4}{|l|}{ Passive smoking } \\
\hline Yes & $295(40.4)$ & $109(43.9)$ & 0.320 \\
\hline No & $436(59.6)$ & $139(56.1)$ & \\
\hline \multicolumn{4}{|c|}{ Pregnancy complication } \\
\hline Yes & $53(6.19)$ & $22(6.0)$ & 0.878 \\
\hline No & $803(93.8)$ & $347(90.0)$ & \\
\hline \multicolumn{4}{|c|}{ History of abortion and stillbirth } \\
\hline Yes & $320(37.4)$ & $147(39.8)$ & 0.271 \\
\hline No & $536(62.6)$ & $222(60.2)$ & \\
\hline \multicolumn{4}{|l|}{ Parity } \\
\hline 0 & $732(86.1)$ & $296(80.8)$ & 0.020 \\
\hline$\geq 1$ & $118(13.9)$ & $70(19.1)$ & \\
\hline
\end{tabular}

$4000 \mathrm{CNY} /$ month, well educated $(76.46 \%$ college-level education or above), without pregnancy complication (93.8\%), without history of abortion and stillbirth (62.6\%). Approximately $40 \%$ of women were exposed to passive smoking during pregnancy. The distributions of these demographic characteristics were not significantly different between the included and excluded women except parity.

Table 2 presents PFHxS, PFOS, PFOA, PFNA, and PFDA were detected in all maternal plasma samples, while PFUdA, PFDoA and PFTrDA were detected in 
Table 2 PFASs concentrations ( $\mathrm{ng} / \mathrm{mL}$ ) of the included pregnant women $(N=856)$

\begin{tabular}{|c|c|c|c|c|c|c|c|c|}
\hline \multirow[t]{2}{*}{ PFAS } & \multirow[t]{2}{*}{ LOD } & \multirow{2}{*}{$\begin{array}{l}>\operatorname{LOD}(\mathrm{N} \\
\%)\end{array}$} & \multirow{2}{*}{$\begin{array}{l}\text { GM } \\
\text { (GSD) }\end{array}$} & \multicolumn{5}{|c|}{ Percentiles } \\
\hline & & & & 5 th & 25th & 50th & 75th & 95th \\
\hline PFHXS & 0.015 & $856(100)$ & $2.7(1.5)$ & 1.3 & 2.1 & 2.8 & 3.6 & 5.6 \\
\hline PFOS & 0.02 & $856(100)$ & $10.7(1.7)$ & 4.2 & 7.6 & 10.7 & 15.7 & 25.6 \\
\hline PFOA & 0.01 & $856(100)$ & 19.9 (1.6) & 9.3 & 15.5 & 20.2 & 27.0 & 38.8 \\
\hline PFNA & 0.02 & $856(100)$ & $1.8(1.6)$ & 0.8 & 1.3 & 1.8 & 2.5 & 4.0 \\
\hline PFDA & 0.01 & $856(100)$ & $2.0(1.9)$ & 0.7 & 1.3 & 2.0 & 3.2 & 6.2 \\
\hline FUdA & 0.01 & $855(99.9)$ & $1.6(2.0)$ & 0.5 & 1.0 & 1.5 & 2.54 & 4.5 \\
\hline PFDoA & 0.015 & 776 (90.6) & $0.1(2.2)$ & LOD & 0.1 & 0.1 & 0.2 & 0.4 \\
\hline PFTrDA & 0.02 & $745(87.0)$ & $0.1(2.1)$ & LOD & 0.1 & 0.1 & 0.2 & 0.4 \\
\hline
\end{tabular}

Note: LOD limit of detection, GM geometric mean, GSD geometric

standard deviation

about 90\% samples. PFOA and PFOS had the highest concentrations (PFOA: $G M=20.2 \mathrm{ng} / \mathrm{mL}$; PFOS: $G M=$ $10.8 \mathrm{ng} / \mathrm{mL})$, followed by PFHxS $(G M=2.7 \mathrm{ng} / \mathrm{mL})$, PFDA $(2.2 \mathrm{ng} / \mathrm{mL})$, PFNA $(1.8 \mathrm{ng} / \mathrm{mL})$, and PFUdA $(1.6$ $\mathrm{ng} / \mathrm{mL}$ ), while PFDoA and PFTrDA had the lowest concentrations.

Table 3 presents the concentrations of FPG and $1 \mathrm{~h}-\mathrm{PG}$ according to the demographic characteristics of the subjects. The mean (SD) FPG and $1 \mathrm{~h}-\mathrm{PG}$ concentrations were $4.04(0.45) \mathrm{mmol} / \mathrm{L}$ (i.e., $72.7(8.1) \mathrm{mg} / \mathrm{dL}$ ) and 6.46 (1.37) $\mathrm{mmol} / \mathrm{L}$ (i.e., $116.3(24.7) \mathrm{mg} / \mathrm{dL}$ ), respectively. The concentrations of FPG and $1 \mathrm{~h}-\mathrm{PG}$ were comparable across pregnant women with different BMI, household income, passive smoking status, pregnancy complication and history of abortion and stillbirth. The concentration of $1 \mathrm{~h}$-PG was higher in pregnant women who were older or had higher education levels, but not in those with FPG. The concentration of FPG was lower in nulliparous pregnant women, but not in those with $1 \mathrm{~h}-\mathrm{PG}$.

Table 4 presents that higher concentrations of PFOS, PFOA, PFNA, PFDA, PFDoA, and PFTrDA were associated with an increased risk of high FPG; however, the associations were not statistically significant $\left(\mathrm{AOR}_{\mathrm{PFOS}}=1.28,95 \% \mathrm{CI}\right.$ : 0.85-1.93; $\mathrm{AOR}_{\mathrm{PFOA}}=1.31,95 \% \mathrm{CI}: 0.77-2.22 ; \mathrm{AOR}_{\mathrm{PFNA}}=$

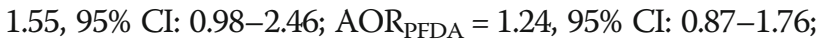
$\mathrm{AOR}_{\text {PFDoA }}=1.09,95 \% \mathrm{CI}: 0.87-1.37 ; \mathrm{AOR}_{\mathrm{PFT} \text { TDA }}=1.12,95 \%$ CI: 0.89-1.40). Higher concentrations of PFASs were associated with an increased risk of high $1 \mathrm{~h}-\mathrm{PG}$, except for PFHxS, and the associations with PFOS, PFNA, PFDA, PFUdA, and PFDoA were statistically significant after adjustment for potential confounders $\left(\mathrm{AOR}_{\mathrm{PFOS}}=1.87,95 \% \mathrm{CI}\right.$ : $1.15-3.05$; $\mathrm{AOR}_{\mathrm{PFNA}}=2.15,95 \% \mathrm{CI}: 1.24-3.74 ; \mathrm{AOR}_{\mathrm{PFDA}}=1.61,95 \%$ CI: $1.10-2.44 ; A_{\text {PFUdA }}=1.71,95 \%$ CI: 1.12-2.62; AOR $_{\text {PF- }}$ DoA $=1.34$, 95\% CI: 1.00-1.81). In addition, multiple linear regressions were also used to analyze the association between PFASs and plasma glucose. Similar results were found in multiple linear regression as in logistic regression model, although the association of PFDoA with $1 \mathrm{~h}-\mathrm{PG}$ is not statistically significant (Supplemental Table S2).

We further examined the associations between the categorized PFAS concentrations and FPG/1 h-PG. Weak associations between the highest tertiles of PFASs and an increased risks of high FPG were observed, but the associations were not statistically significant (Fig. 2). Compared with pregnant women with the lowest tertiles of PFASs, the risk of high $1 \mathrm{~h}-\mathrm{PG}$ was increased in women with the highest tertiles of PFASs, with statistically significant associations observed for PFOS, PFNA, PFDA, PFUdA, and PFDoA $\left(\mathrm{AOR}_{\mathrm{PFOS}}=2.28,95 \% \mathrm{CI}: 1.09-4.75 ; \mathrm{AOR}_{\mathrm{PFNA}}=\right.$ 2.27, 95\% CI: 1.11-4.66; $\mathrm{AOR}_{\mathrm{PFDA}}=2.37$, 95\% CI: $1.18-$ 4.73; $\mathrm{AOR}_{\mathrm{PFUdA}}=2.87,95 \% \mathrm{CI}: 1.25-5.61 ; \mathrm{AOR}_{\mathrm{PFDoA}}=$ 2.52, 95\% CI: 1.21-5.26) (Fig. 3). Linear trends were observed between the tertiles of PFOS, PFNA, PFDA, PFUdA, and PFDoA, and high $1 \mathrm{~h}-\mathrm{PG}$ (P for trend $=0.026$, $0.019,0.011,0.011$, and 0.012 , respectively).

We repeated the analysis after excluding women with GDM. The pattern of associations between PFASs and high FPG and $1 \mathrm{~h}$-PG did not change substantially, except that the association between PFDoA and high $1 \mathrm{~h}$ PG was no longer statistically significant (Supplemental Table S3). In addition, the analysis among pregnant women with a BMI of $<25 \mathrm{~kg} / \mathrm{m}^{2}$ produced similar results (Supplemental Table S4).

In the subgroup analysis for different GW spans, the associations between PFASs and high FPG remained non-significant disregard of the timing of FPG measurements, except that the increased concentrations of PFNA were associated with an increased risk of high FPG at 15-20 GWs $\left(\mathrm{AOR}_{\mathrm{PFNA}}=2.54\right.$, 95\% CI: $1.28-$ 5.07). The pattern of association between PFASs and high $1 \mathrm{~h}-\mathrm{PG}$ did not substantially change, disregard of measurement time of $1 \mathrm{~h}-\mathrm{PG}$, with the exception that the association with high $1 \mathrm{~h}-\mathrm{PG}$ became non-significant for PFOS, PFUdA, PFDoA (at 20-23 GWs), and PFOS, PFNA, PFDA and PFUdA (at 24-28 GWs), largely owing to the reduced sample size (Supplemental Table S5).

\section{Discussion}

In this prospective cohort study, PFAS exposures in pregnant women were found to be associated with high $1 \mathrm{~h}-\mathrm{PG}$, but not FPG, and the association persisted for pregnant women without GDM or with BMI $<25 \mathrm{~kg} / \mathrm{m}^{2}$.

Many studies have demonstrated that PFASs were associated with impaired glucose homeostasis and an increased risk of diabetes in the general population [1719]. However, in pregnant women, the associations between PFASs and glucose homeostasis have not been well investigated. Wang et al's study showed that several PFAS compounds were associated with increased postpartum FPG, including perfluoro-1-metylheptylsulfonat (1 m-PFOS), perfluoro-3/4-metylheptylsulfonat $(3 \mathrm{~m}+4$ 
Table 3 The distribution of FPG and 1 h-PG (mmol/L) according to participant's demographic characteristics

\begin{tabular}{|c|c|c|c|c|}
\hline \multirow[t]{2}{*}{ Characteristics } & \multicolumn{2}{|c|}{$\mathrm{FPG}(N=856)$} & \multicolumn{2}{|c|}{$1 \mathrm{~h}-\mathrm{PG}(\mathrm{N}=705)$} \\
\hline & $\overline{M e a n} \pm$ SD & $P$-value & Mean \pm SD & $P$-value \\
\hline Total & $4.04 \pm 0.45$ & & $6.46 \pm 1.37$ & \\
\hline Maternal age at enrollment (years) & & 0.675 & & $<0.001$ \\
\hline$<25$ & $4.01 \pm 0.39$ & & $5.99 \pm 1.28$ & \\
\hline $25-35$ & $4.04 \pm 0.45$ & & $6.54 \pm 1.35^{*}$ & \\
\hline$\geq 35$ & $4.08 \pm 0.49$ & & $6.73 \pm 1.70^{*}$ & \\
\hline Pre-pregnancy BMI $\left(\mathrm{kg} / \mathrm{m}^{2}\right)$ & & 0.854 & & 0.097 \\
\hline$<18.5$ & $4.06 \pm 0.47$ & & $6.25 \pm 1.38$ & \\
\hline $18.5-24.9$ & $4.04 \pm 0.44$ & & $6.52 \pm 1.36$ & \\
\hline$\geq 25$ & $4.09 \pm 0.45$ & & $6.65 \pm 1.43$ & \\
\hline Maternal education & & 0.077 & & 0.032 \\
\hline Below high school & $4.15 \pm 0.44$ & & $6.05 \pm 1.55$ & \\
\hline High School & $4.05 \pm 0.46$ & & $6.41 \pm 1.54$ & \\
\hline College or above & $4.02 \pm 0.44$ & & $6.52 \pm 1.30^{*}$ & \\
\hline Per capita household income (CNY) & & 0.341 & & 0.702 \\
\hline$<4000$ & $4.05 \pm 0.39$ & & $6.46 \pm 1.33$ & \\
\hline $4000-8000$ & $4.05 \pm 0.46$ & & $6.49 \pm 1.37$ & \\
\hline$>8000$ & $3.99 \pm 0.47$ & & $6.37 \pm 1.39$ & \\
\hline Passive smoking & & 0.512 & & 0.803 \\
\hline Yes & $4.07 \pm 0.47$ & & $6.44 \pm 1.40$ & \\
\hline No & $4.05 \pm 0.43$ & & $6.47 \pm 1.38$ & \\
\hline Pregnancy complication & & 0.725 & & 0.146 \\
\hline Yes & $4.28 \pm 0.43$ & & $6.76 \pm 1.54$ & \\
\hline No & $4.04 \pm 0.45$ & & $6.45 \pm 1.39$ & \\
\hline History of abortion and stillbirth & & 0.156 & & 0.426 \\
\hline Yes & $4.25 \pm 0.43$ & & $6.52 \pm 1.5$ & \\
\hline No & $4.07+0.43$ & & $6.44+1.33$ & \\
\hline Parity & & 0.003 & & 0.059 \\
\hline 0 & $4.07 \pm 0.48$ & & $6.43 \pm 1.36$ & \\
\hline$\geq 1$ & $4.15 \pm 0.43$ & & $6.72 \pm 1.61$ & \\
\hline
\end{tabular}

$F P G$ fasting plasma glucose, $1 \mathrm{~h}-P G 1 \mathrm{~h}$-plasma glucose after a $50-\mathrm{g}$ oral glucose tolerance test

${ }^{*}, p<0.05$, compared with the first group

m-PFOS), perfluoro-5-metylheptylsulfonat (5 m-PFOS), and PFHxS [26]. The Longitudinal Investigation of Fertility and the Environment (LIFE) study reported that each SD increment in PFOA concentrations was associated with a 1.87-fold increase in GDM risk [21]. In the Odense Child Cohort study, in metabolically vulnerable pregnant women (i.e., BMI $\geq 27 \mathrm{~kg} / \mathrm{m}^{2}$, family history of diabetes mellitus, previous GDM, multiple pregnancy, or delivery of a macrosomic child), PFHxS and PFNA concentrations were associated with impaired glycemic status, however, no associations were found in women with low GDM risk [20]. It's a pity that the absence of information on history of family diabetes and subjects with previous GDM limited our ability of examining the association in subjects with high risk. Higher concentrations of PFASs in our study may partially contribute to the differences with other studies. In our study, concentrations of most PFASs were much higher than those in the Odense Child Cohort [20], the LIFE Study [21] and Wang et al' study [26] except that PFOS is higher in the LIFE Study compared to the current study. The differences in concentrations, as well as outcome indices of impaired glucose homeostasis, timing of measurement and population included make the comparison between these studies difficult; nevertheless, the potential for PFAS exposure to disturb glucose homeostasis has been supported in most studies. 
Table 4 Association between PFAS concentrations (In-transformed) and high FPG and 1 h-PG in pregnant women

\begin{tabular}{|c|c|c|c|c|}
\hline \multirow{2}{*}{$\begin{array}{l}\text { In- } \\
\text { PFAS } \\
\text { (ng/ } \\
\mathrm{ml})\end{array}$} & \multicolumn{2}{|l|}{$\mathrm{FPG}(N=856)$} & \multicolumn{2}{|l|}{$1 \mathrm{~h}-\mathrm{PG}(\mathrm{N}=705)$} \\
\hline & COR (95\% Cl) & AOR $(95 \% \mathrm{Cl})$ & COR $(95 \%$ Cl) & AOR (95\% Cl) \\
\hline$\overline{\mathrm{PFH} \times \mathrm{S}}$ & $1.00(0.61,1.62)$ & $0.89(0.51,1.55)$ & $0.92(0.52,1.63)$ & $0.92(0.45,1.86)$ \\
\hline PFOS & $1.28(0.88,1.85)$ & $1.28(0.85,1.93)$ & $1.77(1.15,2.72)$ & $1.87(1.15,3.05)$ \\
\hline PFOA & $1.39(0.86,2.26)$ & $1.31(0.77,2.22)$ & $1.15(0.67,1.92)$ & $1.40(0.75,2.59)$ \\
\hline PFNA & $1.54(1.02,2.34)$ & $1.55(0.98,2.46)$ & $1.74(1.08,2.78)$ & $2.15(1.24,3.74)$ \\
\hline PFDA & $1.25(0.91,1.71)$ & $1.24(0.87,1.76)$ & $1.52(1.06,2.17)$ & $1.61(1.10,2.44)$ \\
\hline PFUdA & $0.99(0.73,1.34)$ & $0.98(0.71,1.35)$ & $1.48(1.04,2.12)$ & $1.71(1.12,2.62)$ \\
\hline PFDoA & $1.13(0.92,1.39)$ & $1.09(0.87,1.37)$ & $1.28(1.00,1.65)$ & $1.34(1.00,1.81)$ \\
\hline PFTrDA & $1.04(0.85,1.28)$ & $1.12(0.89,1.40)$ & $1.12(0.88,1.41)$ & $1.13(0.87,1.49)$ \\
\hline
\end{tabular}

COR crude odds ratio, $A O R$ adjusted odds ratio, $\mathrm{Cl}$ confidence interval, $F P G$ fasting plasma glucose, $1 \mathrm{~h}$-PG $1 \mathrm{~h}$-plasma glucose after a 50 -g oral glucose tolerance test

Models were adjusted for maternal age at enrollment (years), pre-pregnancy BMI $\left(\mathrm{kg} / \mathrm{m}^{2}\right)$, per capita household income, education level, passive smoking, pregnancy complication, history of abortion and stillbirth, and parity

Although the underlying mechanism linking PFASs to glucose homeostasis is not yet clear, it has been suggested that inhibition of phosphorylation of protein kinase B (Akt) and the activation of peroxisome proliferator activated receptors (PPARs) may play a role [10, 27, 28]. Studies using animal models and HepG2 cells have indicated that PFAS compounds reduce the expression of the phosphatase and tensin homolog protein and affect the Akt signaling pathway $[10,29]$. The inhibition of Akt, a key mediator of cellular insulin sensitivity, may stimulate gluconeogenesis and hepatic insulin resistance [29]. Both PFOA and PFOS have been certified to affect glucose metabolism by AKT signaling pathway. However, the other PFASs were not investigated in these studies $[10,29]$. In addition, studies have demonstrated that PFASs can bind to and activate the PPAR $\alpha$ and $\gamma$ receptors [27]. PPAR, a nuclear transcription receptor, is known to play essential roles in the regulation of gene expression, glucose homeostasis, fatty acid metabolism, and inflammation [30]. Therefore, PFAS-activated PPAR could disturb glucose homeostasis by influencing insulin resistance [28] and insulin secretion [31]. PFOA have the highest potential of PPAR $\alpha$ activation than the other PFASs with a shorter carbon chain length (including PFHxS, PFNA, PFDA and PFDoA) [32]. Moreover, PFAS exposure may interfere with secretion and function of glucocorticoids and thyroid hormones via hypothalamic-pituitary-adrenal axis and hypothalamic-pituitary-thyroid axis, which may further disturb glucose metabolism [11, 33, 34]. The physiological effects of PFASs on glycemic homeostasis may depend on the potency and concentration of individual PFASs $[28,32]$.

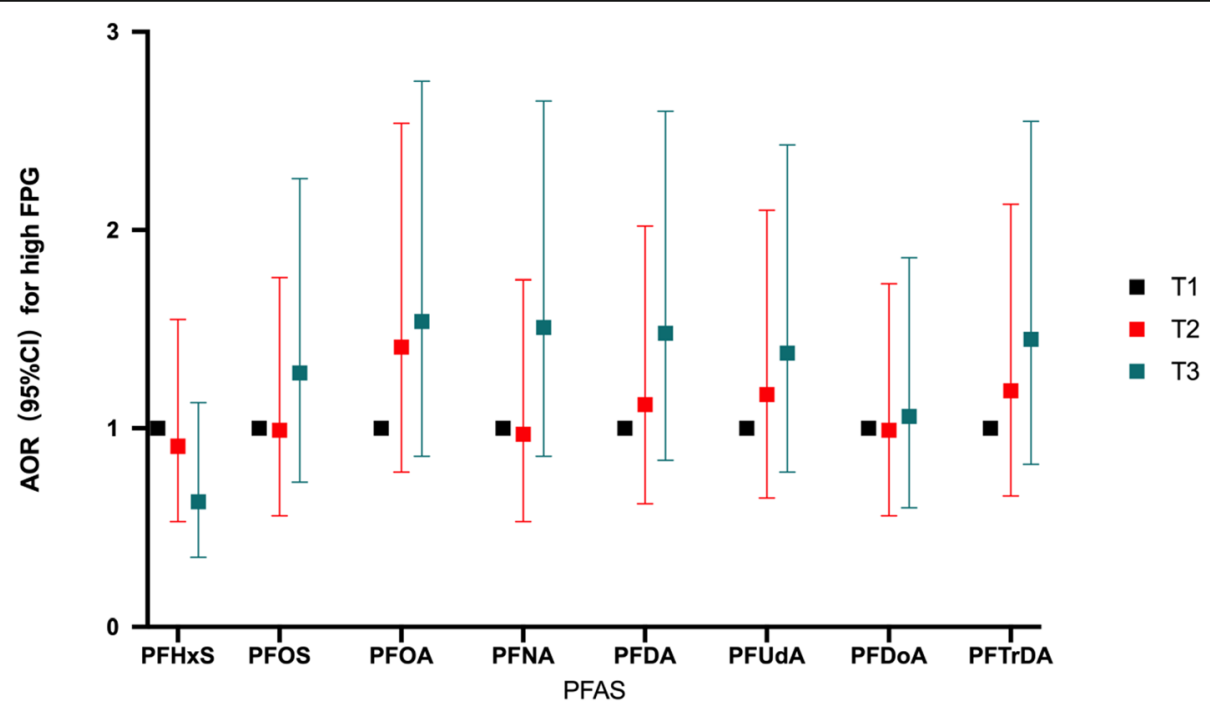

Fig. 2 Association between PFAS concentrations (divided by tertiles) and high FPG. Notes: All the $p$-trend values for PFASs with FPG were insignificant 


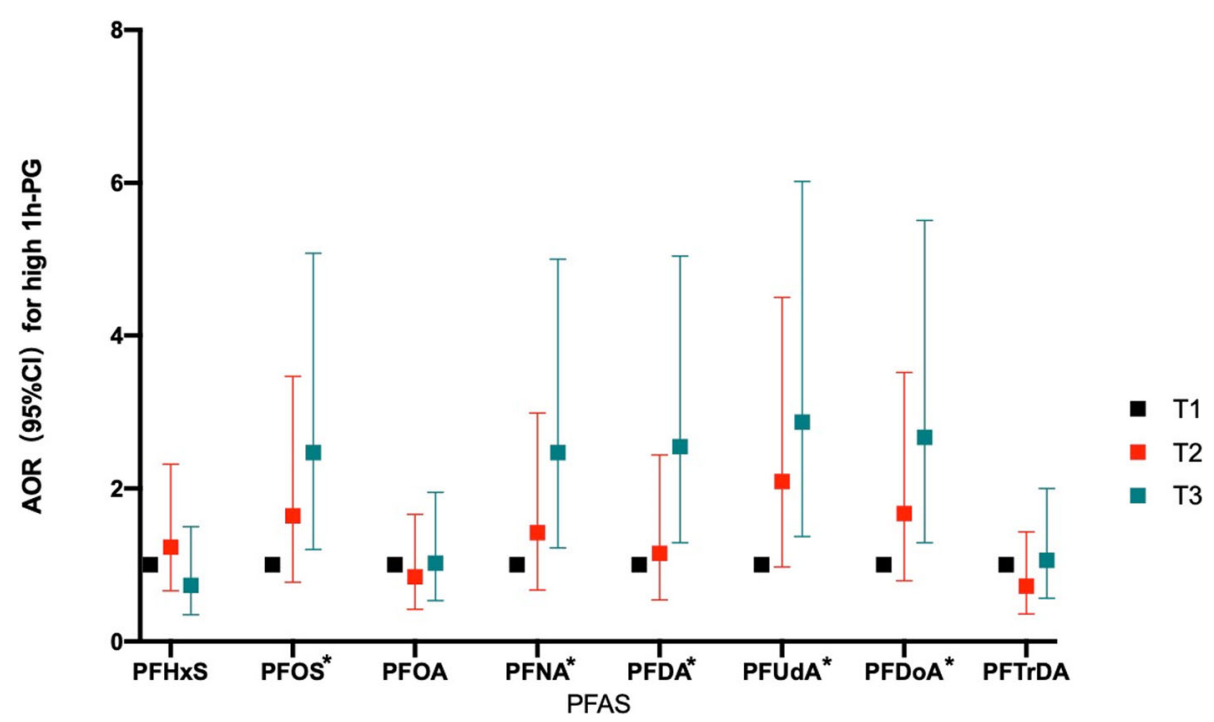

Fig. 3 Association between PFAS concentrations (divided by tertiles) and high $1 \mathrm{~h}-\mathrm{PG}$. Notes: ${ }^{*} p$ for trend $<0.05$

The strengths of the present study were the prospective nature of the study design, the large sample size, and the measurement of a wide range of PFAS compounds. However, the potential limitations of the study should be considered. First, a considerable proportion of subjects was lost to follow-up, which may have led to selection bias. However, the characteristics of the included subjects were similar to those excluded in terms of age, education, pre-pregnancy BMI, and household income, and thus a substantial selection bias was not expected. Second, the follow-up period from the measurement of PFAS exposure to the endpoints (FPG and $1 \mathrm{~h}-\mathrm{PG}$ ) was short, but the single-point measurement of PFAS concentration may reflect PFAS exposure long before the date of blood collection owing to their long half-life. Third, the relationships between PFASs and blood glucose measures may have been confounded by unmeasured confounders, such as family diabetes history and dietary habits; this should be examined in future studies. In addition, information on maternal active smoking was not collected, since the proportion of active smoking was quite low in Chinese women [35]. For example, only $0.4 \%$ of pregnant women have been exposed to active smoking during pregnancy in a Shanghai Birth Cohort [36]. Thus, the current result is not expected to be severely biased by the un-adjustment of active smoking. Fourth, not all the subjects had information on $1 \mathrm{~h}$ PG after the 50-g OGTT, which may have led to missed cases of GDM and affect the association between PFASs and outcome indices in the sensitivity analysis of pregnant women with GDM. However, the absence of GDM cases, if any, would have attenuated the observed association. Fifth, data for FPG (12-20 GWs) or $1 \mathrm{~h}-\mathrm{PG}$ (20-28 GWs) were collected over a long time span, and thus the associations between PFASs and FPG and $1 \mathrm{~h}$ PG may have been confounded by the gestational week. However, we performed subgroup analyses using different GWs spans at glucose measurement, and found that the results did not change significantly.

\section{Conclusion}

Exposure to certain PFASs (i.e., PFOS, PFNA, PFDA, PFUdA, and PFDoA) was associated with an increased risk of high $1 \mathrm{~h}$-PG among pregnant women. Further studies are needed to clarify the effect of PFASs on gestational glycemic homeostasis and the underlying mechanism.

\section{Supplementary information}

Supplementary information accompanies this paper at https://doi.org/10. 1186/s12940-020-00640-8.

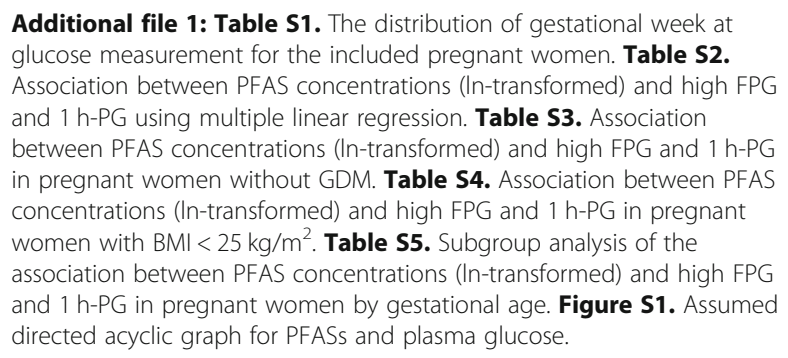

Additional file 1: Table S1. The distribution of gestational week at glucose measurement for the included pregnant women. Table S2. Association between PFAS concentrations (In-transformed) and high FPG and $1 \mathrm{~h}-\mathrm{PG}$ using multiple linear regression. Table S3. Association between PFAS concentrations (In-transformed) and high FPG and 1 h-PG in pregnant women without GDM. Table S4. Association between PFAS concentrations (In-transformed) and high FPG and $1 \mathrm{~h}-\mathrm{PG}$ in pregnant women with $\mathrm{BMl}<25 \mathrm{~kg} / \mathrm{m}^{2}$. Table S5. Subgroup analysis of the association between PFAS concentrations (In-transformed) and high FPG and 1 h-PG in pregnant women by gestational age. Figure S1. Assumed directed acyclic graph for PFASs and plasma glucose.

\section{Abbreviations}

PFASs: Perfluoroalkyl and polyfluoroalkyl substances; FPG: Fasting plasma glucose; 1 h-PG: One-hour plasma glucose; GWs: Gestational weeks; PFHXS: Perfluorohexane sulfonate; PFOS: Perfluorooctane sulfonate; PFOA: Perfluorooctanoic acid; PFNA: Perfluorononanoic acid; PFDA: Perfluorodecanoic acid; PFUdA: Perfluoroundecanoic acid; PFDoA: Perfluorododecanoic acid; PFTrDA: Perfluorotridecanoic acid; ORs: Odds ratios; Cls: Confidence intervals; GDM: Gestational diabetes mellitus; OGTT: Oral glucose tolerance test; S-MBCS: Shanghai-Minhang Birth 
Cohort Study; LOD: Limit of detection; SD: Standard deviations; HOMAIR: Homeostasis model of assessment for insulin resistance; Akt: Protein kinase B; PPARs: Peroxisome proliferator activated receptors

\section{Acknowledgements}

The authors thank fieldworkers involved in the survey for their efforts in data collection and quality control and all the pregnant women investigated.

\section{Authors' contributions}

WY, HL and MM conceived and designed the study. YR, LJ and MM performed data analysis and drafted the article. WY, MM, YR, FY, HL, XS, ZZ and JD revised the manuscript and critically discussed the results. All authors were involved in interpreting the data and approved the final article.

\section{Funding}

This work was supported by grants from the National key research and development program [grant numbers 2016YFC1000505, 2018YFC1002801]; Shanghai Municipal Commission of Health and Family Planning [grant number 201840184]; Innovation-oriented Science and Technology Grant from NHC Key Laboratory of Reproduction Regulation [grant numbers CX2017-06]; and Shandong Medical and Health Science and Technology Development Project [grant numbers 2018WS060].

\section{Availability of data and materials}

The datasets used during the current study are available from the corresponding author on reasonable request.

\section{Ethics approval and consent to participate}

The study was approved by the ethical review committee of Shanghai Institute of Planned Parenthood Research (SIPPR). Written informed consent was obtained before the data collection and analysis, and the survey was conducted in accordance with the Declaration of Helsinki Principles.

\section{Consent for publication}

Not applicable.

\section{Competing interests}

The authors declare they have no actual or potential competing financial interests.

\section{Author details}

'Department of Health Statistics, School of Public Health, Weifang Medical University, Weifang, Shandong, China. ${ }^{2}$ Minhang District Maternal and Child Health Hospital, Shanghai, China. ${ }^{3}$ Department of Global Public Health, Karolinska Institute, Stockholm, Sweden. ${ }^{4} \mathrm{NHC}$ Key Lab of Reproduction Regulation (Shanghai Institute of Planned Parenthood Research), Fudan University, Shanghai, China.

Received: 18 May 2020 Accepted: 6 August 2020

Published online: 17 August 2020

\section{References}

1. Lau C, Anitole K, Hodes C, Lai D, Pfahles-Hutchens A, Seed J. Perfluoroalkyl acids: a review of monitoring and toxicological findings. Toxicol Sci. 2007; 99(2):366-94.

2. Tittlemier SA, Pepper K, Seymour C, Moisey J, Bronson R, Cao XL, Dabeka RW. Dietary exposure of Canadians to perfluorinated carboxylates and perfluorooctane sulfonate via consumption of meat, fish, fast foods, and food items prepared in their packaging. J Agric Food Chem. 2007:55(8): 3203-10.

3. Conder JM, Hoke RA, De Wolf W, Russell MH, Buck RC. Are PFCAs bioaccumulative? A critical review and comparison with regulatory criteria and persistent lipophilic compounds. Environ Sci Technol. 2008;42(4):995-1003.

4. Olsen GW, Burris JM, Ehresman DJ, Froehlich JW, Seacat AM, Butenhoff JL, Zobel LR. Half-life of serum elimination of perfluorooctanesulfonate, perfluorohexanesulfonate, and perfluorooctanoate in retired fluorochemical production workers. Environ Health Perspect. 2007;115(9):1298-305.

5. Bartell SM, Calafat AM, Lyu C, Kato K, Ryan PB, Steenland K. Rate of decline in serum PFOA concentrations after granular activated carbon filtration at two public water systems in Ohio and West Virginia. Environ Health Perspect. 2010;118(2):222-8.
6. Tian Y, Zhou Y, Miao M, Wang Z, Yuan W, Liu X, Wang X, Wang Z, Wen S, Liang $\mathrm{H}$. Determinants of plasma concentrations of perfluoroalkyl and polyfluoroalkyl substances in pregnant women from a birth cohort in Shanghai, China. Environ Int. 2018;119:165-73.

7. Biegel LB, Liu RC, Hurtt ME, Cook JC. Effects of ammonium perfluorooctanoate on Leydig cell function: in vitro, in vivo, and ex vivo studies. Toxicol Appl Pharmacol. 1995;134(1):18-25.

8. Fuentes S, Colomina MT, Rodriguez J, Vicens P, Domingo JL. Interactions in developmental toxicology: concurrent exposure to perfluorooctane sulfonate (PFOS) and stress in pregnant mice. Toxicol Lett. 2006;164(1):81-9.

9. Seacat AM, Thomford PJ, Hansen KJ, Olsen GW, Case MT, Butenhoff JL. Subchronic toxicity studies on perfluorooctanesulfonate potassium salt in cynomolgus monkeys. Toxicol Sci. 2002;68(1):249-64.

10. Yan S, Zhang H, Zheng F, Sheng N, Guo X, Dai J. Perfluorooctanoic acid exposure for 28 days affects glucose homeostasis and induces insulin hypersensitivity in mice. Sci Rep. 2015;5:11029.

11. Goudarzi H, Araki A, Itoh S, Sasaki S, Miyashita C, Mitsui T, Nakazawa H, Nonomura K, Kishi R. The Association of Prenatal Exposure to Perfluorinated chemicals with glucocorticoid and androgenic hormones in cord blood samples: the Hokkaido study. Environ Health Perspect. 2017;125(1):111-8.

12. Barry V, Winquist A, Steenland K. Perfluorooctanoic acid (PFOA) exposures and incident cancers among adults living near a chemical plant. Environ Health Perspect. 2013;121(11-12):1313-8.

13. Lind PM, Salihovic S, van Bavel B, Lind L. Circulating levels of perfluoroalkyl substances (PFASs) and carotid artery atherosclerosis. Environ Res. 2017;152: 157-64.

14. Darrow LA, Stein CR, Steenland K. Serum perfluorooctanoic acid and perfluorooctane sulfonate concentrations in relation to birth outcomes in the mid-Ohio Valley, 2005-2010. Environ Health Perspect. 2013;121(10):1207-13.

15. Matilla-Santander N, Valvi D, Lopez-Espinosa MJ, Manzano-Salgado CB, Ballester F, Ibarluzea J, Santa-Marina L, Schettgen T, Guxens M, Sunyer J, et al. Exposure to Perfluoroalkyl substances and metabolic outcomes in pregnant women: evidence from the Spanish INMA birth cohorts. Environ Health Perspect. 2017:125(11):117004.

16. Zeng XW, Lodge CJ, Dharmage SC, Bloom MS, Yu Y, Yang M, Chu C, Li QQ, Hu LW, Liu KK, et al. Isomers of per- and polyfluoroalkyl substances and uric acid in adults: Isomers of C8 Health Project in China. Environ Int. 2019; 133(Pt A):105160.

17. Lin CY, Chen PC, Lin YC, Lin LY. Association among serum perfluoroalkyl chemicals, glucose homeostasis, and metabolic syndrome in adolescents and adults. Diabetes Care. 2009;32(4):702-7.

18. Cardenas A, Gold DR, Hauser R, Kleinman KP, Hivert MF, Calafat AM, Ye X, Webster TF, Horton ES, Oken E. Plasma concentrations of per- and Polyfluoroalkyl substances at baseline and associations with glycemic indicators and diabetes incidence among high-risk adults in the diabetes prevention program trial. Environ Health Perspect. 2017;125(10):107001.

19. Su TC, Kuo CC, Hwang JJ, Lien GW, Chen MF, Chen PC. Serum perfluorinated chemicals, glucose homeostasis and the risk of diabetes in working-aged Taiwanese adults. Environ Int. 2016:88:15-22.

20. Jensen RC, Glintborg D, Timmermann CAG, Nielsen F, Kyhl HB, Andersen HR, Grandjean P, Jensen TK, Andersen M. Perfluoroalkyl substances and glycemic status in pregnant Danish women: the Odense child cohort. Environ Int. 2018;116:101-7.

21. Zhang C, Sundaram R, Maisog J, Calafat AM, Barr DB, Buck Louis GM. A prospective study of prepregnancy serum concentrations of perfluorochemicals and the risk of gestational diabetes. Fertil Steril. 2015; 103(1):184-9.

22. Valvi D, Oulhote Y, Weihe P, Dalgard C, Bjerve KS, Steuerwald U, Grandjean P. Gestational diabetes and offspring birth size at elevated environmental pollutant exposures. Environ Int. 2017;107:205-15.

23. Metzger BE, Gabbe SG, Persson B, Buchanan TA, Catalano PA, Damm P, Dyer AR, Leiva A, Hod M, Kitzmiler JL, et al. International association of diabetes and pregnancy study groups recommendations on the diagnosis and classification of hyperglycemia in pregnancy. Diabetes Care. 2010;33(3):676-82

24. consultation We. Appropriate body-mass index for Asian populations and its implications for policy and intervention strategies. Lancet. 2004;363(9403): 157-63.

25. Lee KW, Ching SM, Ramachandran V, Yee A, Hoo FK, Chia YC, Wan Sulaiman WA, Suppiah S, Mohamed MH, Veettil SK. Prevalence and risk factors of gestational diabetes mellitus in Asia: a systematic review and meta-analysis. BMC Pregnancy Childbirth. 2018;18(1):494. 
26. Wang Y, Zhang L, Teng Y, Zhang J, Yang L, Li J, Lai J, Zhao Y, Wu Y. Association of serum levels of perfluoroalkyl substances with gestational diabetes mellitus and postpartum blood glucose. J Environ Sci (China). 2018:69:5-11.

27. Vanden Heuvel JP, Thompson JT, Frame SR, Gillies PJ. Differential activation of nuclear receptors by perfluorinated fatty acid analogs and natural fatty acids: a comparison of human, mouse, and rat peroxisome proliferatoractivated receptor-alpha, -beta, and -gamma, liver $\mathrm{X}$ receptor-beta, and retinoid X receptor-alpha. Toxicol Sci. 2006;92(2):476-89.

28. Fleisch AF, Rifas-Shiman SL, Mora AM, Calafat AM, Ye X, Luttmann-Gibson H, Gillman MW, Oken E, Sagiv SK. Early-life exposure to Perfluoroalkyl substances and childhood metabolic function. Environ Health Perspect. 2017;125(3):481-7.

29. Qiu T, Chen M, Sun X, Cao J, Feng C, Li D, Wu W, Jiang L, Yao X. Perfluorooctane sulfonate-induced insulin resistance is mediated by protein kinase B pathway. Biochem Biophys Res Commun. 2016;477(4):781-5.

30. Pyper SR, Viswakarma N, Yu S, Reddy JK. PPARalpha: energy combustion, hypolipidemia, inflammation and cancer. Nucl Recept Signal. 2010;8:e002.

31. Lupi R, Del Guerra S, Marselli L, Bugliani M, Boggi U, Mosca F, Marchetti P, Del Prato $\mathrm{S}$. Rosiglitazone prevents the impairment of human islet function induced by fatty acids: evidence for a role of PPARgamma2 in the modulation of insulin secretion. Am J Physiol Endocrinol Metab. 2004;286(4): E560-7.

32. Buhrke T, Kibellus A, Lampen A. In vitro toxicological characterization of perfluorinated carboxylic acids with different carbon chain lengths. Toxicol Lett. 2013;218(2):97-104.

33. Pereiro N, Moyano R, Blanco A, Lafuente A. Regulation of corticosterone secretion is modified by PFOS exposure at different levels of the hypothalamic-pituitary-adrenal axis in adult male rats. Toxicol Lett. 2014; 230(2):252-62.

34. Long M, Ghisari M, Bonefeld-Jørgensen EC. Effects of perfluoroalkyl acids on the function of the thyroid hormone and the aryl hydrocarbon receptor. Environ Sci Pollut Res Int. 2013;20(11):8045-56

35. Wang Y, MA S, Shen Z, Feng Y, Wu S, Yan B, Cai S, Ma L, Jiang Y. Association of tobacco exposure with first trimester depression. Chin J Prev Control Chron Dis. 2019;27(7):484-7.

36. Wang WJ, Zhang L, Zhang DL, Zheng T, He H, Fang F, Zhang J, Ouyang F, Luo ZC. Exploring Fetal Sex Dimorphism in the Risk Factors of Gestational Diabetes Mellitus-A Prospective Cohort Study. Front Endocrinol (Lausanne). 2019;10:848

\section{Publisher's Note}

Springer Nature remains neutral with regard to jurisdictional claims in published maps and institutional affiliations.

Ready to submit your research? Choose BMC and benefit from:

- fast, convenient online submission

- thorough peer review by experienced researchers in your field

- rapid publication on acceptance

- support for research data, including large and complex data types

- gold Open Access which fosters wider collaboration and increased citations

- maximum visibility for your research: over $100 \mathrm{M}$ website views per year

At $\mathrm{BMC}$, research is always in progress.

Learn more biomedcentral.com/submissions 